At the end of that time the plants in the air chamber were markedly healthier and bigger than those in the hydrogen chamber.

A similar experiment was afterwards set up, observing the same precautions, but it was modified to yield quantitative data and to give some indication of the effect of hydrogen on the growth of the plant. Bell jars as gas chambers sufficiently large to cover forty tubes were used. A third chamber was added, through which a gas mixture of, in percentage proportions, hydrogen 40 , nitrogen 40 , oxygen 20 , and carbon dioxide $0 \cdot 03$, was drawn. This experiment was kept going for eight weeks, and at the end of that period the lengths of all the leaves and the dry weights on each plant were determined. The lengths of the leaves were measured as indices of area; the small width of the leaves rendered the direct determination of this quantity extremely difficult. The mean total leaf length in centimetres and the mean dry weight in grams per plant are presented in the accompanying table.

\begin{tabular}{l|c|lc}
\hline \multicolumn{1}{c|}{ Gas Mix ture } & Culture Solution & & \\
\hline $\begin{array}{l}\text { Nitrogen, } \\
\text { oxygen and } \\
\text { carbon dioxide }\end{array}$ & + Nitrate & $\begin{array}{l}\text { Leaf length } \\
\text { Dry weight }\end{array}$ & $\begin{array}{c}26 \cdot 4 \\
0 \cdot 0018\end{array}$ \\
\hline $\begin{array}{l}\text { Nitrogen, } \\
\text { hydrogen, } \\
\text { oxygen and } \\
\text { carbon dioxide }\end{array}$ & - Nitrate & $\begin{array}{l}\text { Leaf length } \\
\text { Dry weight }\end{array}$ & $\begin{array}{c}21 \cdot 5 \\
0 \cdot 00148(2)\end{array}$ \\
\hline $\begin{array}{l}\text { Hydrogen, } \\
\text { oxygen and } \\
\text { carbon dioxide }\end{array}$ & - Nitrate & $\begin{array}{l}\text { Leaf length } \\
\text { Dry weight }\end{array}$ & $\begin{array}{l}36 \\
0 \cdot 00322(3)\end{array}$ \\
\hline
\end{tabular}

The difference between the dry weights of (2) and (6) is only slightly greater than the standard error of the difference of these means, but the difference in leaf lengths is more than twice the standard error of the difference of the means. The latter difference is, therefore, probably significant. The best growth was that made in the gas mixture containing both nitrogen and hydrogen. The stimulating effect of hydrogen under the conditions of the experiment is also evident from series (5) when compared against series (1). 'The conclusions would therefore seem to be justifiable that the stimulating effect of hydrogen is masking the depressant effect of absence of nitrogen in (5) and (6).

Botanical Dept.,

R. Brown.

Seale-Hayne Agricultural College, Newton Abbot.

\section{Ambiguity in Sign of Spearman's General Factor}

IN a previous letter, ${ }^{1}$ I gave a sketch of a method for obtaining an explicit formula for the general factor $g$ and the specific factors which occur in Spearman's two-factor theory of intelligence. By developing this method, I obtain the following results.

If the tetrad relations hold, the factors exist. If two more conditions hold, the factors are real. If a fourth condition holds, they are unique except in sign. In the case which occurs most often in actual experiment, when all the coefficients of correlation between the tests are positive, the value of the general factor with one sign seems to correspond to something like general ability and the value with the other sign to the lack of that ability. But in other cases, when some of the coefficients of correlation are negative, it is not clear how to distinguish between the interpretations of the two possible values of the general factor.

After a discussion of the effects of pooling tests, the results have been applied to a dice problem, and to actual sets of mental tests. It is found that both for the dice throws and for the mental tests, the general factor exists and is real, but is not unique, even in numerical value. The comparison between dice throws and mental tests, when examined closely, breaks down in one important respect. Finally, the possibility is examined of making the numerical value of the general factor approximately unique, by making the ratio of the standard deviations of the indeterminate and determinate parts a given small fraction. I have shown that to do this by merely multiplying the number of tests is quite impracticable; the only method available is to choose one or more tests that correlate very highly indeed with $g$. In the case of Kelley's tests, it is found that the fraction which we wish to make small appears to be $0 \cdot 09$, but that this value is very doubtful on account of the tetrad relations not being satisfied exactly. The use of figures corrected for attenuation leads to the conclusion that either some of the factors are imaginary, or, what is more probable, that the correction for attenuation is not reliable.

A full account of this work will be offered to a psychological journal in the near future.

University College,

H. T. H. Piaggio.

\section{Nottingham.} Dec. 21.

1 "The General Factor in Spearman's Theory of Intelligence," NATURE, 127, 56, Jan. 10, 1931.

\section{Spectra of Bromine: Br V, VII and IV}

IN continuation of our previous work on the spectra of arsenic and selenium, the spectrum of bromine has been investigated by a study of photographs taken under different conditions of excitation. The chief multiplets belonging to the higher spark spectra of bromine have been easily identified. The characteristic intervals of the deepest ${ }^{2} P$ terms in $\mathrm{Br} V$ and $\mathrm{Br}$ VII have been found to be $6090 \mathrm{~cm} .^{-1}$ and $7580 \mathrm{~cm} .{ }^{-1}$ respectively, while $4 p{ }^{3} P_{0}-4 p{ }^{3} P_{1}$ and $4{ }^{3} P_{1}-4 p{ }^{3} P_{2}$ of $\mathrm{Br} \mathrm{V}$ are 2115 and 4906 units.

A full report of the analysis will be published shortly.

The experimental data referred to above have led one of us (A.S.R.) further to the identification of many of the chief groups of Br IV arising from the transition of the electron from the $4 p$ to the $5 s$, $4 d$ and $s p^{3}$ states. The intervals of $5 s{ }^{3} P_{0,1,2}$ are found to be $1281 \mathrm{~cm}^{-1}$ and $4688 \mathrm{~cm}^{-1}$. This classification does not agree with the scheme suggested by $\mathrm{S}$. C. Deb ${ }^{1}$, which the writer thinks is out of step in the iso-electronic sequence Ge I to Br IV.

Science College,

A. S. RAO.

Andhra University,

K. R. RAo.

Waltair, Nov. 1.

${ }^{1}$ Proc. Roy. Soc., A, 12y, 197 ; 1930. 